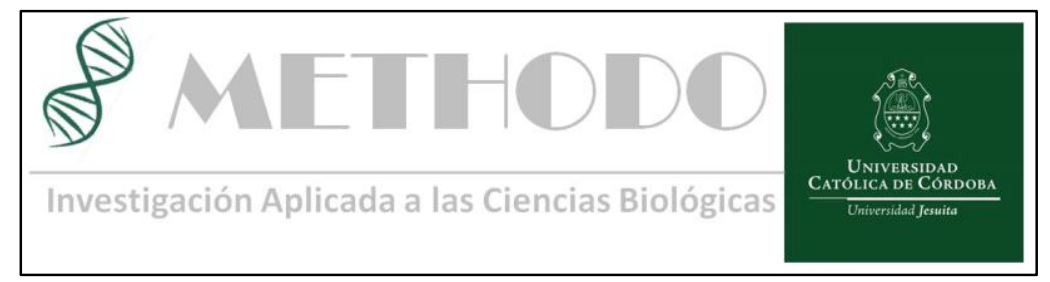

CASO CLINICO Methodo 2018;3(3):78-82

DOI: 10.22529/me.2018.3(3)06

Recibido 30 Jun 2018 | Aceptado 06 Ago 2018 | Publicado 13 Sep 2018

\title{
La nanotecnología aplicada al desarrollo de resinas compuestas y sistemas adhesivos a esmalte y dentina. Reporte de un caso clínico.
}

\section{Nanotechnology applied to the development of resin composite and adhesive systems for enamel and dentin. A case report.}

\author{
Tomás Calza, Od. ${ }^{1}$, Ana Carolina Carranza Astrada, Od. ${ }^{1}$, Claudia Estela Bonnin, Prof. Dra. ${ }^{1}$ \\ ${ }^{1}$ Docentes de las cátedras de Técnica y Clínica de Operatoria Dental, Carrera de Odontología, Facultad de Ciencias de la Salud, \\ Universidad Católica de Córdoba. Caso clínico realizado con la colaboración del alumno de Clínica de \\ Operatoria Dental Santiago Bioletto.
}

\section{Resumen}

La incorporación de nanopartículas a la fase inorgánica de las resinas compuestas procura mejorar propiedades mecánicas y ópticas, aumentando el porcentaje de relleno y reduciendo consecuentemente los fenómenos de contracción de polimerización. El caso clínico que se presenta refleja alguna de las indicaciones en las cuales se aplican estas innovaciones tecnológicas.

\begin{abstract}
Incorporation of nanosized fillers in the inorganic phase of resin composites aims at improving their mechanical and optical properties, increasing the proportion of ceramic particles thus reducing the polymerization shrinkage. The case reported in this article reflects one of the indications of these enhanced materials, where technological innovations are applied.
\end{abstract}

\section{Introducción:}

Como ocurre en todos los campos de la ciencia y la tecnología, la Odontología ha experimentado cambios vertiginosos en los últimos tiempos, que han aportado nuevos y valiosos recursos en el diagnóstico, tratamiento y prevención de las afecciones que tienen asiento en el sistema estomatognático. Uno de estos avances tiene que ver con los aportes de la nanotecnología en las diferentes especialidades odontológicas.
El término "nano" deriva de la palabra griega para "enano"1, y de acuerdo al Diccionario de la Lengua Española, significa la milmillonésima parte de algo $^{2}$. Así, en el sistema métrico, un nanómetro comprende la milmillonésima parte del metro $\left(0,000000001 \mathrm{~m}\right.$ o $\left.10-^{9} \mathrm{~m}\right)$, y como unidad de longitud, se suele utilizar para expresar dimensiones a un nivel atómico. El diámetro de un átomo de hidrógeno, por ejemplo, es de $0,074 \mathrm{~nm}^{3}$. Se le atribuye al premio Nobel de Física Richard P. Feynman, en su conferencia titulada "Hay mucho espacio en el fondo" de 1959, el nacimiento del concepto de nanotecnología ${ }^{4-7}$ La Iniciativa Nacional sobre Nanotecnología de Estados Unidos define a ésta como la manipulación de la materia en la que al menos una dimensión posee un tamaño entre 1 y 100 nanómetros ${ }^{3-9}$. Puede también definirse como el arte y la ciencia de la ingeniería de materiales a una escala menor a 100 nm5. Así descripta, la nanotecnología ha revolucionado el terreno de la Medicina y de la Odontología, ya que ha permitido desarrollos en la ciencia de los materiales y en la biotecnología que condujeron a la producción de materiales con mejores propiedades físicas y mecánicas ${ }^{3-5}$

\section{Resinas compuestas}

Las resinas compuestas de uso odontológico son materiales formados por una matriz orgánica basada en diferentes tipos de resinas sintéticas, 
más un material de relleno como por ejemplo la sílice. La matriz es la responsable del endurecimiento del material, mientras que el relleno afecta las propiedades físico-mecánicas del material polimerizado. Un agente de enlace a base de silano mantiene ambas fases unidas. Estos materiales tienen aplicaciones en prácticamente todas las especialidades odontológicas, incluyendo la restauración de elementos dentarios, y su empleo involucra técnicas de adhesión al esmalte y a la dentina, mediante sistemas adhesivos específicos también basados en resinas ${ }^{3-6}$. Las resinas compuestas desplazaron un material emblemático de la Odontología Restauradora que prestó servicio durante más de un siglo, la amalgama dental. En buena medida, dicha evolución tuvo que ver con el desarrollo de los rellenos céramicos, que permitieron la aparición gradual de materiales de uso universal en todos los sectores de la cavidad bucal. Los composites de microrellenos originales adolecían de la resistencia necesaria para su uso en las zonas sometidas al estrés de la oclusión, por lo que sólo podían emplearse en la restauración de los elementos dentarios anteriores ${ }^{3}$. El paso de los años permitió el desarrollo de materiales de elevada proporción de relleno en el volumen total del material, lo que les confiere la resistencia necesaria para su uso en zonas de gran exigencia oclusal (sector de premolares y molares), y a su vez con un tamaño de partículas de relleno cada vez menor, que aporta a mejores características ópticas del material (mejor pulido). En este último aspecto es dónde interviene la nanotecnología.

Los nanocomposites han sido una de las mayores contribuciones a la Odontología restauradora y estética de los últimos tiempos para la restauración de la estructura dental. Estos materiales se caracterizan por presentar partículas de relleno con un tamaño menor a los $100 \mathrm{~nm}$, que ofrecen ventajas estéticas y de resistencia frente a las resinas compuestas de microrrelleno e híbridas. Presentan mejores características de pulido, lo que produce superficies más suaves respecto a las de los composites con tamaños de partículas mayores. El uso de partículas de relleno cada vez menores resulta en una mejor dispersión y en una mayor área interfacial entre matriz y relleno, que se traduce en mayor resistencia flexural y microdureza superficial ${ }^{3}$. Módulo de elasticidad superior, reducción en la contracción de polimerización y mejores características de manipulación son otras ventajas reportadas ${ }^{1-8}$. Partículas de nanoclusters tan pequeñas como 2-20 $\mathrm{nm}$ son utilizadas actualmente en los composites restauradores de nanorrellenos, con su potencial en las propiedades ópticas del material y sus ventajas en la Odontología estética (los tonos translúcidos presentan tamaños de relleno de entre 5 y $20 \mathrm{~nm}$ ).
Las mejores propiedades ópticas se deben a que estas partículas de relleno presentan un tamaño menor a la longitud de onda de la luz visible (400 a $800 \mathrm{~nm}$ ), por lo que no reflejan la luz, y por tanto su translucidez se ve aumentada ${ }^{3}$.

Uno de los grandes desafíos actuales en el desarrollo de estos materiales con nanopartículas, es lograr la fabricación de partículas discretas no aglomeradas que puedan ser distribuidas uniformemente en la matriz resinosa, ya que la aglomeración de las nanopartículas convierte al material en uno de microrrelleno ${ }^{3}$.

Las aplicaciones de la nanotecnología no se limitan sólo a producir mejores rellenos para los composites, sino también intervienen desarrollo de materiales "inteligentes" con propiedades preventivas. Se ha reportado el reciente desarrollo de un composite en cuyo relleno se incorporan partículas de fosfato de calcio nano-amorfo recargable, que no sólo mejoran las propiedades remineralizantes del material, sino que también le confiere la capacidad de mantener constantes sus niveles de calcio y fosfato a través de recarga y liberación. Los investigadores lo describieron como un material "inteligente" por su habilidad constante para neutralizar rápidamente los ácidos bacterianos a lo largo de los márgenes de las restauraciones por medio de la liberación de calcio y fosfato, y por tanto con capacidad de inhibir la iniciación de caries secundaria ${ }^{5}$.

Un intento adicional por lograr capacidad en los materiales restauradores de prevenir el inicio de caries secundaria fue la aplicación de un recubrimiento de nanocomposite compuesto por chitosan modificado con lactosa (Chitlac) con nanopartículas de plata. Las nanopartículas se encuentran uniformemente distribuidas y son capaces de reducir significativamente la formación de biofilm en la superficie de la restauración en un $80 \%$ luego de $48 \mathrm{~h}$ de la aplicación, en comparación al control tratado sin dicho recubrimiento. Las imágenes obtenidas con microscopía láser confocal de barrido revelaron que, si bien el recubrimiento no tenía propiedades bactericidas, poseía la habilidad de alterar la morfología del biofilm, inhibiendo el desarrollo de especies maduras en el mismo ${ }^{5}$.

Por último, se reportó que la incorporación de nanopartículas de polietilenimina cuaternaria entrecruzada a las resinas compuestas también ofrece efectos antibacterianos sobre varios patógenos orales, incluyendo Enterococofecalis, Estreptococo mutans, Actinomycesviscosus y Lactobacilo casei. La incorporación de estas nanopartículas causa la interrupción del intercambio iónico a través de las membranas bacterianas del biofilm, llevando a la muerte celular. Estas propiedades bactericidas tal vez lleven a la aparición de una segunda generación de 
materiales restauradores, con una incidencia de fallas por caries secundaria significativamente menor 5 .

\section{Sistemas adhesivos}

Como se explicará más arriba, las resinas compuestas son adheridas a los tejidos dentales duros por medio de sistemas adhesivos específicos, que son también basados en resinas. Se trata de materiales de elevada fluidez, con capacidad de penetrar desde la superficie del esmalte y la dentina adecuadamente tratados, para trabarse micromecánicamente allí luego de su polimerización. La naturaleza de dicho proceso es diferente en el esmalte y en la dentina, pero el resultado en ambos casos es permitir la adhesión de las resinas compuestas restauradoras a tales tejidos.

En principio, estas resinas no poseían carga inorgánica de relleno, pero con el devenir de los años surgió la idea de aplicarles una carga cerámica para incrementar su resistencia, y como una manera de disminuir su contracción de polimerización, que provocaba problemas de desprendimiento de las paredes cavitarias y de sensibilidad posoperatoria. Los primeros sistemas adhesivos en incorporar rellenos en su composición utilizaron las mismas partículas presentes en las resinas compuestas, lo que les confería un mayor espesor de película. Esto permitía no sólo disminuir la contracción de polimerización, sino también actuar como una capa que permite aliviar las tensiones generadas durante la polimerización de la resina compuesta. A su vez, la presencia de relleno mejora sus propiedades mecánicas ${ }^{11}$.

El aumento en la viscosidad y disminución del escurrimiento de un sistema adhesivo, merced a la incorporación de carga inorgánica, podría obstaculizar la capacidad de penetración del material en la dentina acondicionada para formar la capa híbrida, perdiendo capacidad de adaptación y sellado marginal. En este aspecto, la incorporación de nanorrellenos permite trabajar con partículas con un tamaño promedio de $5 \mathrm{~nm}$, y este diminuto tamaño en el relleno permite superar el problema de penetración antes mencionado, y conservando la menor contracción de polimerización y propiedades mecánicas superiores respecto a los adhesivos sin relleno. No obstante, su menor espesor de película no les permite actuar como amortiguadores de la contracción de polimerización de las resinas compuestas $^{11}$.

Ciertas marcas comerciales incorporan $10 \%$ en peso de partículas esféricas de sílice de $5 \mathrm{~nm}$ de diámetro en sus sistemas adhesivos, a través de un proceso que evita la aglomeración de estas para formar clusters. Así, estas partículas extremadamente pequeñas se mantienen en suspensión coloidal sin precipitar, lo que asegura al profesional que el material se encuentra listo para ser utilizado en todo momento, sin tener que agitarlo previo a su dispensado ${ }^{8}$.

Azad y col. ${ }^{10}$ encontraron mayor resistencia flexural y adhesiva en un adhesivo experimental que incorporaba 0,2 a $0,5 \%$ en peso de nanopartículas de sílice silanizada.

\section{Caso clínico}

A continuación, se presenta el caso de una paciente de 37 años, con una restauración próximo-oclusal provisoria en un segundo premolar inferior (figura 1), que requiere reemplazo por una restauración definitiva.

Luego del aislamiento absoluto del campo operatorio y de la adaptación del sistema de matriz y cuña (figura 2), se realizó el acondicionamiento ácido simultáneo del esmalte y de la dentina (grabado total) con un gel de ácido fosfórico al $25 \%$ (figura 3 ).

Se utilizaron en la restauración tres materiales con nanotecnología de reciente aparición en nuestro medio, todos de la casa DentsplySirona. El sistema adhesivo, denominado XP Bond ${ }^{\mathrm{MR}}$, es un adhesivo universal de grabado total, que presenta un nanorrelleno de partículas de sílice amorfa ${ }^{12}$ (figura 4).

Las resinas compuestas utilizadas fueron dos: en primer lugar, un composite fluido de inserción en bloque (Surefil SDR Flow ${ }^{\mathrm{MR}}$ ), que permite colocar incrementos de hasta $4 \mathrm{~mm}$ de espesor para realizar rellenos, especialmente indicado para la restauración de este tipo de preparaciones cavitarias. Este material presenta un relleno inorgánico de un 47,3\% en volumen, con un tamaño de partícula que oscila entre $20 \mathrm{~nm}$ y 10 $\mu \mathrm{m}$. Se trata, por tanto, de un nanohíbrido (figura 5). En segundo lugar, en superficie, se utilizó un composite restaurador nanohíbrido de alta carga cerámica (Spectra Smart ${ }^{\mathrm{MR}}$ ), de uso universal en los sectores anterior y posterior de la cavidad bucal $^{12}$ (figura 6). Luego de la fotopolimerización (figura 7), se realizó el pulido final de la restauración (figura 8). La (figura 9) muestra la restauración terminada. 


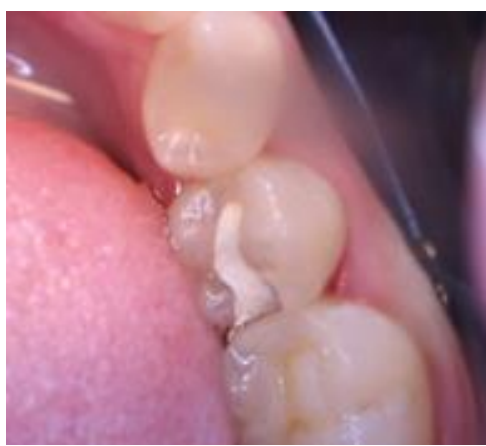

Figura 1.

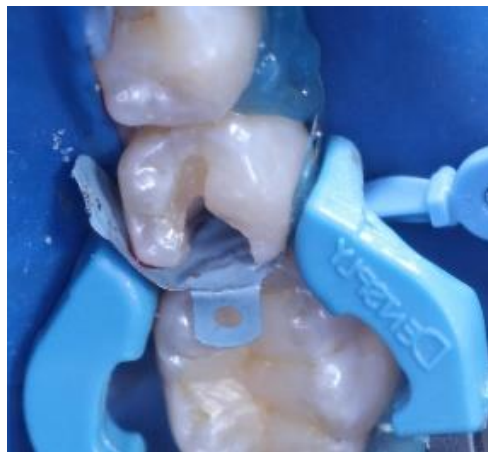

Figura 4.

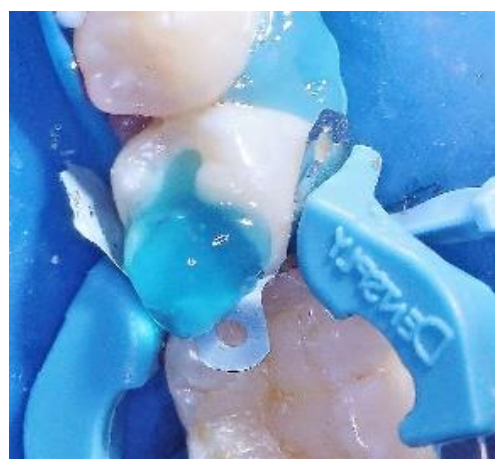

Figura 7.

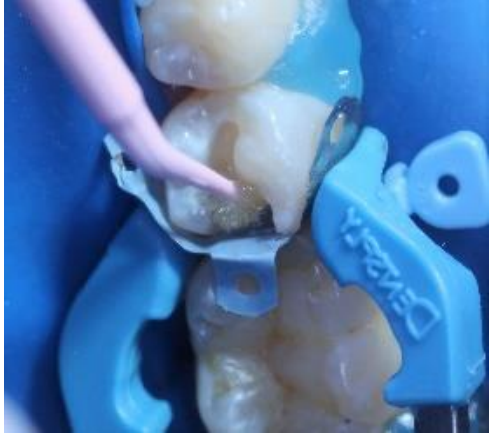

Figura 2.

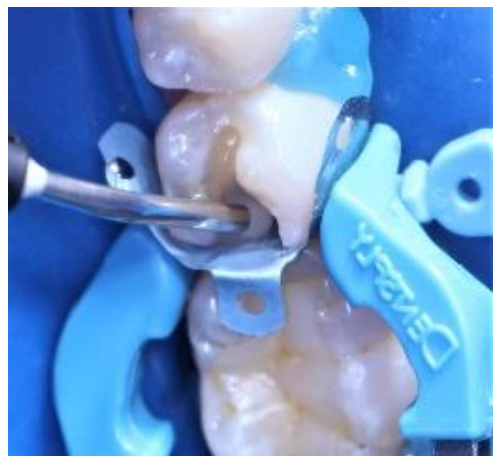

Figura 5.

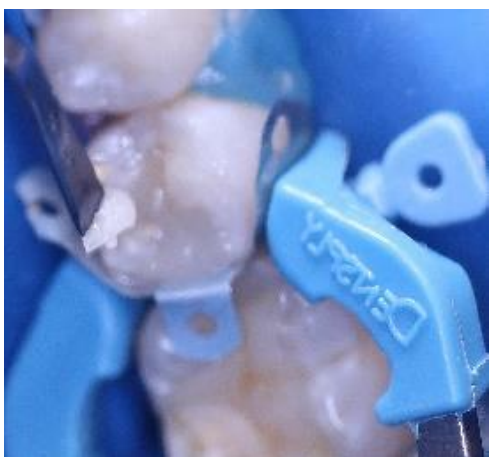

Figura 8.

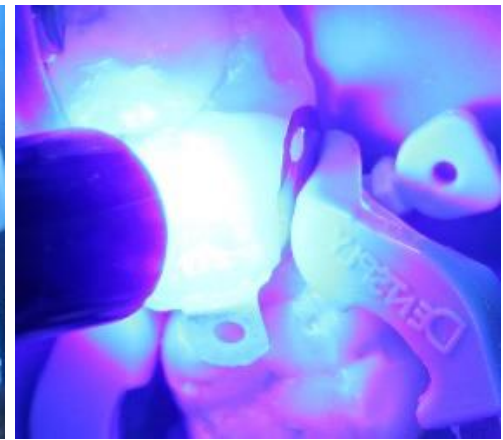

Figura 3.

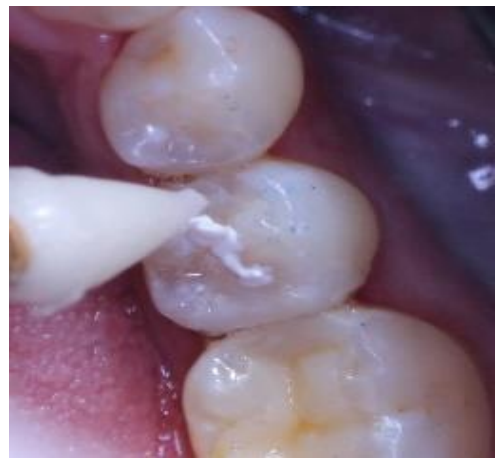

Figura 6.

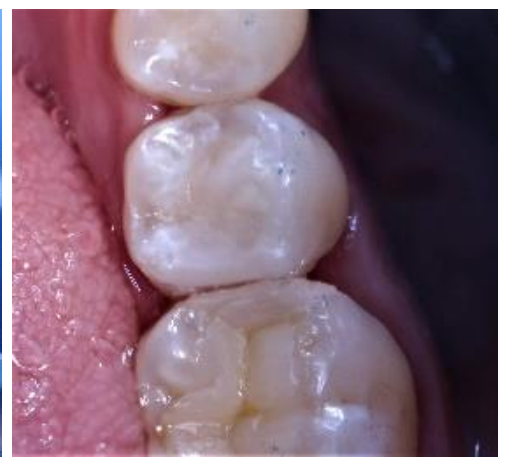

Figura 9.

Figura 1 a 9: 1. Restauración provisoria inicial. 2. Aislamiento absoluto del campo operatorio y adaptación de matriz y cuña para la reconstrucción de la cara proximal. 3. Acondicionamiento ácido simultáneo de esmalte y dentina. 4. Aplicación del adhesivo XP BondMR. 5. Aplicación del composite Surefil SDR Flow+MR. 6. Últimos incrementos realizados con Spectra SmartMR. 7. Fotopolimerización.

8. Pulido. 9. Restauración terminada. 


\section{Bibliografía:}

1. Saravana Kumar R, Vijayalakshmi R. Nanotechnology in dentistry. Ind J Dent Res 2006; 17(2):62-65.

2. www.dle.rae.es

3. Ogle OE, Byles N. Nanotechnology in dentistry today. West Indian Med J 2014; 63(4):344-48. DOI: 10.7727/wimj.2013.178.

4. Freitas RA. Nanodentistry. JADA 2000; 131:1559-65.

5. Alkahtani RN. The implications and applications of nanotechnology in dentistry: a review. Saudi Dental Journal 2018; 30:107116.

6. Rickman L J, Padipatvuthikul P, Chee B. Clinical applications of preheated hybrid resin composite. Br Dent J 2011; 211(2):63-67.

7. Sharan J, Singh S, Lale SV, Mishra M, Koul V, Kharbanda OP. Applications of nanomaterials in dental science: a review. $\mathrm{J}$ NanosciNanotechnol 2017; 17(4):2235-55.

8. Jhaveri HM, Balaji PR. Nanotechnology: the future of dentistry. J IndProsthodSoc 2005; 5(1):15-17.

9. Cantín M, Vilos C, Suazo I. Nanodentistry: the future of Dentistry based on nanotechnology systems. Int $\mathbf{J}$ Odontostomat 2010; 4(2):127-32.

10. Azad E, Atai M, Zandi M, Shokrollahi P, Solhi L. Structure-properties relationships in dental adhesives: effect of initiator, matrix monomer structure, and nano-filler incorporation. Dent Mater 2018, https://doi.org/10.1016/j.dental.2018.05.013.

11. Bertoldi Hepburn A. Nanotecnología en la formulación de nuevos composites. http://www.odontologos.com.co

12. http://www.dentsplyargentina.com.ar

\section{Palabras claves:}

RESINAS COMPUESTAS, NANOTECNOLOGÍA, RESTAURACIONES DENTALES

\section{Keywords:}

RESIN COMPOSITE, NANOTECHNOLOGY, DENTAL RESTORATIONS

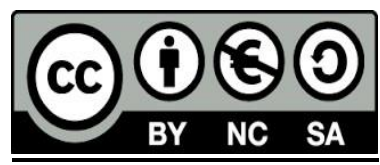

